\title{
Electrical control of quantum-dot fine-structure splitting for high-fidelity hole spin initialization
}

\author{
J. D. Mar,,${ }^{1,2, *}$ J. J. Baumberg, ${ }^{2}$ X. L. Xu, ${ }^{1, \dagger}$ A. C. Irvine, ${ }^{3}$ and D. A. Williams ${ }^{1}$ \\ ${ }^{1}$ Hitachi Cambridge Laboratory, Cavendish Laboratory, Cambridge CB3 OHE, United Kingdom \\ ${ }^{2}$ NanoPhotonics Centre, Cavendish Laboratory, University of Cambridge, Cambridge CB3 OHE, United Kingdom \\ ${ }^{3}$ Microelectronics Research Centre, Cavendish Laboratory, University of Cambridge, Cambridge CB3 OHE, United Kingdom
}

(Received 13 November 2015; revised manuscript received 17 December 2015; published 27 January 2016)

\begin{abstract}
We demonstrate electrical control of the neutral exciton fine-structure splitting in a single InAs/GaAs selfassembled quantum dot by significantly reducing the splitting to near zero through the application of a vertical electric field in the fast electron tunneling regime. This is verified by performing high-resolution photocurrent spectroscopy of the two fine-structure split exciton eigenstates as a function of reverse bias voltage. Using the qubit initialization scheme for a quantum-dot hole spin based on rapid electric-field ionization of a spin-polarized exciton, our results suggest a practical approach towards achieving qubit initialization with near-unity fidelity in the absence of magnetic fields.
\end{abstract}

DOI: 10.1103/PhysRevB.93.045316

A promising candidate for a solid-state qubit is the spin of an electron or hole confined in a semiconductor quantum dot (QD) due to its long relaxation and decoherence times [1-3] and its scalability into large arrays. For the practical implementation of quantum computing based on such qubits, a necessary requirement is the fast initialization of individual spins with high fidelity and within a scalable device architecture [4]. With the belief that hole spins [5-16] are at least as suitable as qubits compared to electron spins [17-21] due to their weak hyperfine interaction with the nuclear spin ensemble and vanishing phonon-coupled spin relaxation in the limit of a zero magnetic field and with the recent demonstration of optical coherent spin manipulation and readout without magnetic fields [22], there has been an increased interest recently [12,23-25] in demonstrating fast high-fidelity hole spin initialization without magnetic fields via rapid electric-field ionization of a spinpolarized neutral exciton $\left(X^{0}\right)$. However, the achieved fidelities using this initialization scheme have so far been limited by an intrinsic $X^{0}$ fine-structure splitting $\delta_{\mathrm{FS}}$ [26], which causes $X^{0}$ spin precession prior to the ionization via electron tunneling.

Here we demonstrate electrical control of the $X^{0}$ finestructure splitting in a single InAs/GaAs self-assembled QD by greatly decreasing $\delta_{\mathrm{FS}}$ to values approaching zero through the application of a vertical electric field in the fast electron tunneling regime. This is confirmed by performing highresolution photocurrent (PC) spectroscopy of the two linearly polarized fine-structure split $X^{0}$ eigenstates as a function of reverse bias voltage using resonant continuous-wave (cw) laser excitation. Compared to other approaches [24,27-30], our results may provide a practical approach towards achieving near-unity fidelity initialization of individual hole spin qubits without magnetic fields through the application of a vertical electric field, which may be realized within a scalable device architecture towards a future quantum computer.

\footnotetext{
*Author to whom correspondence should be addressed: jm585@cam.ac.uk

${ }^{\dagger}$ Present address: Beijing National Laboratory for Condensed Matter Physics, Institute of Physics, Chinese Academy of Sciences, Beijing 100190, People's Republic of China.
}

The device used in this paper was designed for single-QD PC measurements and fabricated as an $n-i$-Schottky photodiode structure based on a two-dimensional electron gas (2DEG). A single layer of InAs/GaAs self-assembled QDs, which was grown to yield a low surface density of QDs $\left(\sim 10^{9} \mathrm{~cm}^{-2}\right)$, was embedded in a 250-nm-thick layer of $i$-GaAs and positioned $50 \mathrm{~nm}$ above a Si $\delta$-doped GaAs layer $\left(N_{d}=5 \times 10^{12} \mathrm{~cm}^{-2}\right)$ from which the $2 \mathrm{DEG}$ is derived and confined in the resultant V-shaped potential well [31,32]. In order to perform single-QD PC measurements, submicrometer-sized apertures were etched into an $\mathrm{Al}$ shadow mask via electron-beam lithography to isolate single QDs. In addition, $\mathrm{Cr} / \mathrm{Au}$ bond pads were formed on both the Schottky contact and the ohmic contact (which was made to the 2DEG) to allow for electrical connection of the device to an external voltage source and current meter. Further details of the device structure can be found in Ref. [12]. Prior to single-QD PC measurements, we first performed biasdependent microphotoluminescence spectroscopy in order to promptly locate isolated single QDs, measure their particular $X^{0}$ transition energy, and determine the bias voltage range within which a single-QD PC signal may be expected as a result of $e-h$ pair ionization and tunneling out from the QD following resonant laser excitation of the $X^{0}$ transition [33,34].

PC spectra of the two $X^{0}$ fine-structure split eigenstates, which are characterized by linear polarizations along the [110] and [1 10] crystallographic axes, are obtained by using linearly polarized cw laser fields aligned with the [110] and [110] axes, respectively. This is achieved by using the method of sweeping the $X^{0}$ transition energy through a fixed laser energy $E_{\text {laser }}^{0}$ via the quantum-confined Stark effect (QCSE) by tuning the bias voltage $V_{b}$ (or vertical electric field $F$ ) across the device [12,34-36]. Such a PC measurement is shown in Fig. 1 for a given QD using $E_{\text {laser }}^{0}=1364.38 \mathrm{meV}$ where the [110] and [1 10$]$ axes are parallel to the laboratory's vertical and horizontal axes, respectively, and the spectra are shifted vertically in the figure with respect to each other for clarity. The broad spectral linewidth of the PC peaks is the result of the fast picosecond-time-scale electron tunneling out of the QD under such high electric fields. By fitting Lorentzian curves to the PC peaks (solid lines), corresponding values of $V_{b}$ and $X^{0}$ transition energies, which are known from the value of $E_{\text {laser }}^{0}$, can be obtained for each linearly polarized 


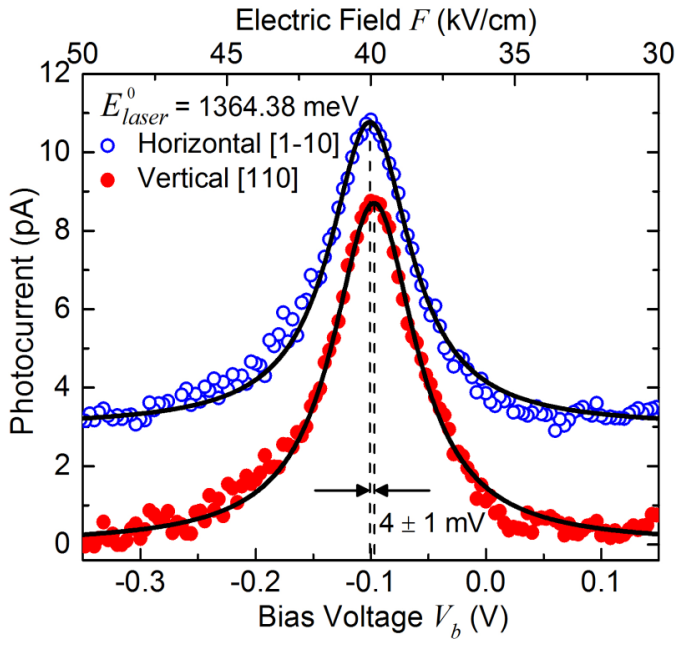

FIG. 1. PC spectra of the two $X^{0}$ fine-structure split eigenstates, which are characterized by linear polarizations along the [110] and [110] crystallographic axes for a given value of $E_{\text {laser }}^{0}$. The [110] and [1110] axes are parallel to the laboratory's vertical and horizontal axes, respectively, and the spectra are shifted vertically in the figure with respect to each other for clarity. By fitting Lorentzian curves to the PC peaks (solid lines), corresponding values for $V_{b}$ and $X^{0}$ transition energies can be obtained for each linearly polarized $X^{0}$ eigenstate.

$X^{0}$ eigenstate. By repeating the above measurements for a series of distinct values of $E_{\text {laser }}^{0}$ throughout the range within which a measurable $X^{0}$ PC peak can be obtained, a set of corresponding values of $V_{b}$ and $X^{0}$ transition energies are produced for each eigenstate. Figure 2 shows pairs of spectra of the $X^{0}$ eigenstates for three distinct values of $E_{\text {laser }}^{0}$, which are indicated in the figure, whereas Fig. 3 is a plot of the set of corresponding values of $V_{b}$ and $X^{0}$ transition energies for each eigenstate throughout the $V_{b}$ range within which a PC peak can be obtained. Due to the QCSE, a quadratic curve of $X^{0}$ transition energy as a function of $V_{b}$, which is given by $E\left(V_{b}\right)=A V_{b}^{2}+B V_{b}+C$, can be fit to the experimental data in Fig. 3 (solid lines), yielding a precise relation between $V_{b}$

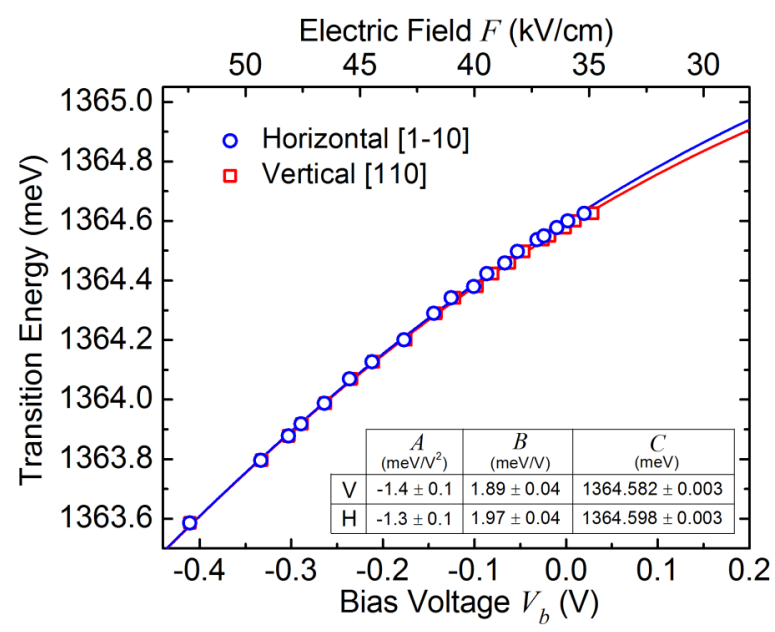

FIG. 3. Plot of the set of corresponding values of $V_{b}$ and $X^{0}$ transition energies for each linearly polarized eigenstate throughout the $V_{b}$ range within which a PC peak can be obtained. Due to the QCSE, a quadratic curve $E\left(V_{b}\right)=A V_{b}^{2}+B V_{b}+C$ can be fit to the experimental data (solid lines), yielding a precise relation between $V_{b}$ and $X^{0}$ transition energies for each eigenstate. The results of such quadratic fits are shown in the table for each eigenstate.

and $X^{0}$ transition energies for each of the linearly polarized eigenstates. The results of such quadratic fits are shown in the table in Fig. 3 for each eigenstate. Therefore, for the $V_{b}$ range within which a PC peak can be measured, $\delta_{\mathrm{FS}}$ as a function of $V_{b}$ is obtained by taking the difference between the quadratic fit curves of the two linearly polarized $X^{0}$ eigenstates as shown by the solid curve in Fig. 4 where a positive value of $\delta_{\mathrm{FS}}$ corresponds to a higher energy for the [1 10 ] horizontally polarized eigenstate. Each data point of $\delta_{\mathrm{FS}}$ in Fig. 4 is obtained by taking the difference between $E_{\text {laser }}^{0}$ for a given spectrum of the [110] eigenstate and the transition energy of the [1 10$]$ eigenstate as derived from its quadratic fit curve at the $V_{b}$ value corresponding to the peak $V_{b}$ of the spectrum of the [110] eigenstate. These values of $\delta_{\mathrm{FS}}$ are reflected in the various
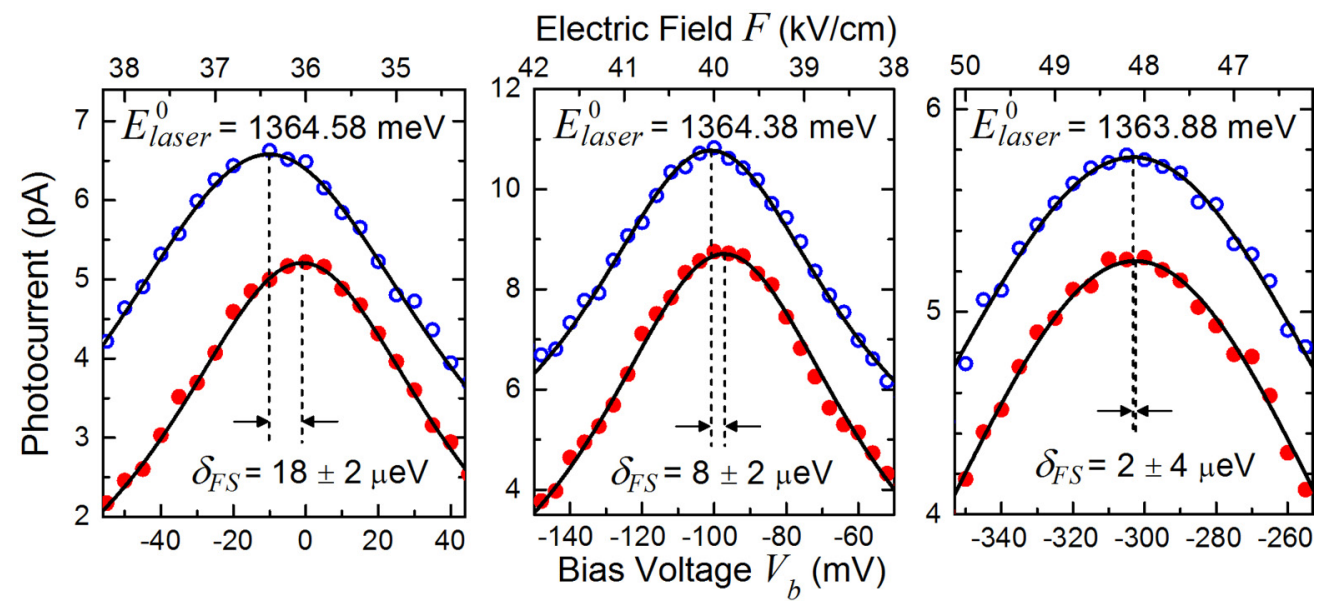

FIG. 2. Pairs of spectra of the $X^{0}$ eigenstates for three distinct values of $E_{\text {laser }}^{0}$ throughout the range within which a measurable $X^{0}$ PC peak can be obtained. By fitting Lorentzian curves to the PC peaks (solid lines), pairs of corresponding values for $V_{b}$ and $X^{0}$ transition energies can be obtained. In each plot of the figure, spectra are shifted vertically with respect to each other for clarity. Values for $\delta_{\mathrm{FS}}$ are indicated in the figure for each pair of spectra and are obtained as described in the text. 


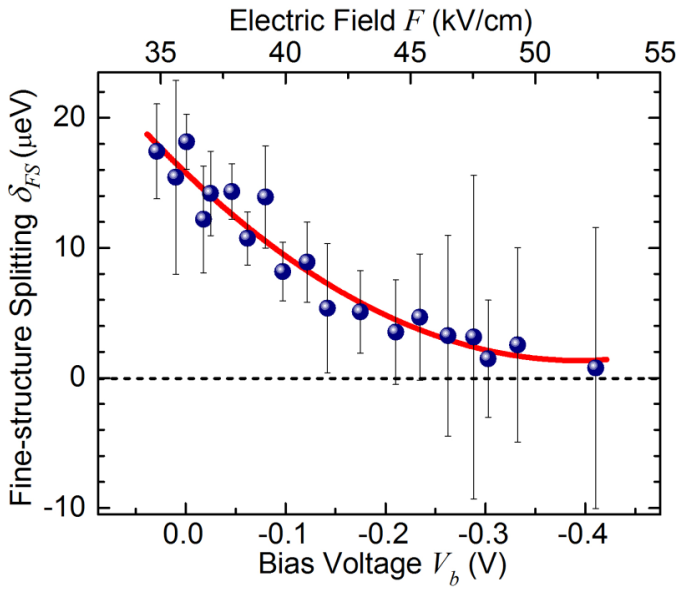

FIG. 4. $X^{0}$ fine-structure splitting energy $\delta_{\mathrm{FS}}$ as a function of $V_{b}$ throughout the $V_{b}$ range within which a PC peak can be measured. The solid curve is obtained by taking the difference between the quadratic fit curves of the two linearly polarized $X^{0}$ eigenstates in Fig. 3. Each data point of $\delta_{\mathrm{FS}}$ is obtained as described in the text.

PC spectra in Fig. 2. As can be seen in Fig. 4, there is a significant reduction of $\delta_{\mathrm{FS}}$ towards near zero with increasing $F$ or reverse bias voltage. For example, $\delta_{\mathrm{FS}}$ decreases from a value of $18 \pm 2 \mu \mathrm{eV}$ at $F=36.0 \mathrm{kV} / \mathrm{cm}\left(V_{b}=-1.0 \mathrm{mV}\right)$ to a value of $2 \pm 4 \mu \mathrm{eV}$ at $F=48.1 \mathrm{kV} / \mathrm{cm}\left(V_{b}=-302.6 \mathrm{mV}\right)$. Furthermore, at $F=52.4 \mathrm{kV} / \mathrm{cm}\left(V_{b}=-410.6 \mathrm{mV}\right), \delta_{\mathrm{FS}}$ is reduced to a value of $0.76 \mu \mathrm{eV}$. Although the error for this data point is relatively large, the clear trend in the experimental data of $\delta_{\mathrm{FS}}$ vs $F$ in Fig. 4 suggests that such near-zero values of $\delta_{\mathrm{FS}}$ are indeed plausible. It should be noted that the experimental data in Fig. 4 cover the entire $V_{b}$ range within which a measurable $X^{0}$ PC peak can be obtained. It should also be noted that the $X^{0}$ eigenstates are constantly aligned with the [110] crystallographic axes throughout this range, thereby eliminating the possibility of coherent coupling of the eigenstates that would be observed as a rotation of the orientation of the eigenstates in the plane of the QD [37]. The observed decrease in $\delta_{\mathrm{FS}}$ with increasing $F$ in Fig. 4 may be the result of an in-plane symmetrizing of the electron and hole wave functions in the QD [38], a vertical spatial separation of the wave functions in the QD [38,39], an in-plane expansion of the exciton being pushed into the barriers that leads to a reduction in the $e-h$ exchange energy [30,40,41], and/or an unknown physical mechanism which requires further investigation. Finally, we note that we have also observed results similar to those of the QD reported here in several other single QDs with similar transition energies in our sample.

We have demonstrated electrical control of the $X^{0}$ finestructure splitting in a single QD by greatly reducing $\delta_{\mathrm{FS}}$ to near zero through the application of a vertical electric field in the fast electron tunneling regime. This was confirmed by performing high-resolution PC spectroscopy of the two fine-structure split eigenstates as a function of reverse bias voltage. Our results suggest a practical approach towards realizing ultrafast initialization of individual hole spin qubits with near-unity fidelity in the absence of magnetic fields. For example, when $F=48.1 \mathrm{kV} / \mathrm{cm}\left(V_{b}=-302.6 \mathrm{mV}\right)$ a theoretical fidelity of $>99.99999 \%$ (or, equivalently, a loss in fidelity of $<10^{-7}$ ) is predicted [42] for the QD used here, while the initialization time and hole lifetime are 0.6 and $474.5 \mathrm{ps}$, respectively. Although the above hole lifetime is relatively short for achieving efficient spin storage [12,25] (which is the reason we refrained from presenting hole spin initialization measurements here), this may be easily resolved by using techniques, such as postgrowth thermal annealing [29], to appropriately reduce the as-grown inhomogeneous $\delta_{\mathrm{FS}}$ of the QD ensemble. This would allow the electric field required to achieve near-zero $\delta_{\mathrm{FS}}$ to be lower where hole lifetimes may be sufficiently long to allow for efficient spin storage while still meeting the requirements for speed in hole spin initialization [12]. Additionally, thermal annealing would cause the QD carrier confinement in the growth direction to become more shallow, further increasing the hole lifetime relative to a constant electron lifetime, which would have shifted to a lower electric field [12]. Thermal annealing, however, does not diminish the necessity to be able to electrically tune $\delta_{\mathrm{FS}}$ to near zero since individual QDs would still have to be independently fine-tuned within the ensemble. Therefore, our results represent an important step towards the practical implementation of ultrafast initialization of individual hole spin qubits with near-unity fidelity within a scalable device architecture.

The authors gratefully acknowledge Dr. S. Nair (University of Toronto) for helpful and contributing discussions and Professor C. R. Stanley (University of Glasgow) for providing the wafers. J.D.M. would like to thank the Higher Education Funding Council for England (HEFCE), the Cambridge Commonwealth Trust (CCT), and the Natural Sciences and Engineering Research Council (NSERC) of Canada for financial support.
[1] D. Loss and D. P. DiVincenzo, Phys. Rev. A 57, 120 (1998).

[2] A. Imamoğlu, D. D. Awschalom, G. Burkard, D. P. DiVincenzo, D. Loss, M. Sherwin, and A. Small, Phys. Rev. Lett. 83, 4204 (1999).

[3] Semiconductor Spintronics and Quantum Computation, edited by D. D. Awschalom, D. Loss, and N. Samarth (Springer, Berlin, 2002).

[4] D. P. DiVincenzo, Fortschr. Phys. 48, 771 (2000).

[5] D. V. Bulaev and D. Loss, Phys. Rev. Lett. 95, 076805 (2005).
[6] J. Fischer, W. A. Coish, D. V. Bulaev, and D. Loss, Phys. Rev. B 78, 155329 (2008).

[7] J. Fischer and D. Loss, Phys. Rev. Lett. 105, 266603 (2010).

[8] B. Eble, C. Testelin, P. Desfonds, F. Bernardot, A. Balocchi, T. Amand, A. Miard, A. Lemaître, X. Marie, and M. Chamarro, Phys. Rev. Lett. 102, 146601 (2009).

[9] D. Brunner, B. D. Gerardot, P. A. Dalgarno, G. Wüst, K. Karrai, N. G. Stoltz, P. M. Petroff, and R. J. Warburton, Science 325, 70 (2009). 
[10] K. De Greve, P. L. McMahon, D. Press, T. D. Ladd, D. Bisping, C. Schneider, M. Kamp, L. Worschech, S. Höfling, A. Forchel, and Y. Yamamoto, Nat. Phys. 7, 872 (2011).

[11] P. Fallahi, S. T. Yılmaz, and A. Imamoğlu, Phys. Rev. Lett. 105, 257402 (2010).

[12] J. D. Mar, J. J. Baumberg, X. L. Xu, A. C. Irvine, and D. A. Williams, Phys. Rev. B 90, 241303(R) (2014).

[13] A. Greilich, S. G. Carter, D. Kim, A. S. Bracker, and D. Gammon, Nat. Photonics 5, 702 (2011).

[14] J. Houel, J. H. Prechtel, A. V. Kuhlmann, D. Brunner, C. E. Kuklewicz, B. D. Gerardot, N. G. Stoltz, P. M. Petroff, and R. J. Warburton, Phys. Rev. Lett. 112, 107401 (2014).

[15] B. D. Gerardot, D. Brunner, P. A. Dalgarno, P. Öhberg, S. Seidl, M. Kroner, K. Karrai, N. G. Stoltz, P. M. Petroff, and R. J. Warburton, Nature (London) 451, 441 (2008).

[16] D. Heiss, S. Schaeck, H. Huebl, M. Bichler, G. Abstreiter, J. J. Finley, D. V. Bulaev, and D. Loss, Phys. Rev. B 76, 241306(R) (2007).

[17] I. A. Merkulov, Al. L. Efros, and M. Rosen, Phys. Rev. B 65, 205309 (2002).

[18] A. V. Khaetskii, D. Loss, and L. Glazman, Phys. Rev. Lett. 88, 186802 (2002).

[19] A. Greilich, D. R. Yakovlev, A. Shabaev, A. L. Efros, I. A. Yugova, R. Oulton, V. Stavarache, D. Reuter, A. Wieck, and M. Bayer, Science 313, 341 (2006).

[20] D. Press, K. De Greve, P. L. McMahon, T. D. Ladd, B. Friess, C. Schneider, M. Kamp, S. Höfling, A. Forchel, and Y. Yamamoto, Nat. Photonics 4, 367 (2010).

[21] A. Bechtold, D. Rauch, F. Li, T. Simmet, P.-L. Ardelt, A. Regler, K. Müller, N. A. Sinitsyn, and J. J. Finley, Nat. Phys. 11, 1005 (2015).

[22] J. Hansom, C. H. H. Schulte, C. Le Gall, C. Matthiesen, E. Clarke, M. Hugues, J. M. Taylor, and M. Atatüre, Nat. Phys. 10, 725 (2014)

[23] K. Müller, A. Bechtold, C. Ruppert, C. Hautmann, J. S. Wildmann, T. Kaldewey, M. Bichler, H. J. Krenner, G. Abstreiter, M. Betz, and J. J. Finley, Phys. Rev. B 85, 241306(R) (2012).

[24] A. J. Brash, L. M. P. P. Martins, F. Liu, J. H. Quilter, A. J. Ramsay, M. S. Skolnick, and A. M. Fox, Phys. Rev. B 92, 121301(R) (2015).
[25] P.-L. Ardelt, T. Simmet, K. Müller, C. Dory, K. A. Fischer, A. Bechtold, A. Kleinkauf, H. Riedl, and J. J. Finley, Phys. Rev. B 92, 115306 (2015).

[26] M. Bayer, G. Ortner, O. Stern, A. Kuther, A. A. Gorbunov, A Forchel, P. Hawrylak, S. Fafard, K. Hinzer, T. L. Reinecke, S. N. Walck, J. P. Reithmaier, F. Klopf, and F. Schäfer, Phys. Rev. B 65, 195315 (2002).

[27] S. Seidl, M. Kroner, A. Högele, K. Karrai, R. J. Warburton, A. Badolato, and P. M. Petroff, Appl. Phys. Lett. 88, 203113 (2006).

[28] R. Trotta, E. Zallo, C. Ortix, P. Atkinson, J. D. Plumhof, J. van den Brink, A. Rastelli, and O. G. Schmidt, Phys. Rev. Lett. 109, 147401 (2012).

[29] A. Greilich, M. Schwab, T. Berstermann, T. Auer, R. Oulton, D. R. Yakovlev, M. Bayer, V. Stavarache, D. Reuter, and A. Wieck, Phys. Rev. B 73, 045323 (2006).

[30] R. M. Stevenson, R. J. Young, P. See, D. G. Gevaux, K. Cooper, P. Atkinson, I. Farrer, D. A. Ritchie, and A. J. Shields, Phys. Rev. B 73, 033306 (2006).

[31] K. Ploog, J. Cryst. Growth 81, 304 (1987).

[32] L. M. R. Scolfaro, R. P. Camata, J. M. V. Martins, and J. R. Leite, Superlattices Microstruct. 12, 203 (1992).

[33] J. D. Mar, X. L. Xu, J. J. Baumberg, F. S. F. Brossard, A. C. Irvine, C. Stanley, and D. A. Williams, Phys. Rev. B 83, 075306 (2011).

[34] J. D. Mar, X. L. Xu, J. J. Baumberg, A. C. Irvine, C. Stanley, and D. A. Williams, J. Appl. Phys. 110, 053110 (2011).

[35] J. D. Mar, X. L. Xu, J. J. Baumberg, A. C. Irvine, C. Stanley, and D. A. Williams, Appl. Phys. Lett. 99, 031102 (2011).

[36] J. D. Mar, J. J. Baumberg, X. L. Xu, A. C. Irvine, C. R. Stanley, and D. A. Williams, Phys. Rev. B 87, 155315 (2013).

[37] A. J. Bennett, M. A. Pooley, R. M. Stevenson, M. B.Ward, R. B. Patel, A. Boyer de la Giroday, N. Sköld, I. Farrer, C. A. Nicoll, D. A. Ritchie, and A. J. Shields, Nat. Phys. 6, 947 (2010).

[38] E. Kadantsev and P. Hawrylak, Phys. Rev. B 81, 045311 (2010).

[39] G. W. Bryant, N. Malkova, and J. Sims, Phys. Rev. B 88, 161301(R) (2013).

[40] S. Fafard and C. Nì. Allen, Appl. Phys. Lett. 75, 2374 (1999).

[41] R. J. Young, R. M. Stevenson, A. J. Shields, P. Atkinson, K. Cooper, D. A. Ritchie, K. M. Groom, A. I. Tartakovskii, and M. S. Skolnick, Phys. Rev. B 72, 113305 (2005).

[42] T. M. Godden, S. J. Boyle, A. J. Ramsay, A. M. Fox, and M. S. Skolnick, Appl. Phys. Lett. 97, 061113 (2010). 